\title{
rf surface impedance of a two-band superconductor
}

\author{
Binping Xiao* \\ Collider-Accelerator Department, Brookhaven National Laboratory, Upton, New York 11973-5000, USA \\ C. E. Reece \\ Thomas Jefferson National Accelerator Facility, Newport News, Virginia 23606, USA
}

(Received 6 April 2018; published 6 May 2019)

\begin{abstract}
In this paper, we extend the Mattis-Bardeen theory to obtain the surface impedance of a two-band superconductor, and apply it to magnesium diboride $\left(\mathrm{MgB}_{2}\right)$ for radiofrequency (rf) superconductivity applications. The numerical results for $\mathrm{MgB}_{2}$ are in good agreement with the previously published experimental results. The surface impedance properties are clearly dominated by the smaller gap, significantly limiting utility in the $10-20 \mathrm{~K}$ regime that might otherwise have been attractive.
\end{abstract}

DOI: $10.1103 /$ PhysRevAccelBeams.22.053101

\section{INTRODUCTION}

The recent activities of superconducting radiofrequency (SRF) accelerating cavities for the Linac Coherent Light Source II (LCLS-II) particle accelerator made from bulk niobium $(\mathrm{Nb})$ materials are the state-of-art activities for high efficiency continuous wave accelerators for exploring frontier physics. The highest quality factor of single cell cavities was pushed to $7 \times 10^{10}$ at $1.3 \mathrm{GHz}$ and $2 \mathrm{~K}$ temperature [1], essentially fully exploiting the theoretical potential of niobium to accelerating field gradients of at least $20 \mathrm{MV} / \mathrm{m}$ [2]. Alternative superconducting materials with critical temperature and superheating critical field higher than those of $\mathrm{Nb}$ are of great interest as the next opportunity for continued technological progress. $\mathrm{MgB}_{2}$ has been one of the candidate materials under investigation for possible future use in SRF applications since its discovery to be a superconductor by Nagamatsu et al. in 2001 [3]. $\mathrm{MgB}_{2}$ is considered to be a conventional superconductor with a high critical temperature, $T_{c}$, of $\sim 39-40 \mathrm{~K}$ [4]. It has two energy gaps, with a $\pi$-gap at $2.3 \mathrm{meV}$ and a $\sigma$-gap at $7.1 \mathrm{meV}$ [5-8], two corresponding coherence lengths between 1.6 and $5.0 \mathrm{~nm}$ and between 3.7 and $12.8 \mathrm{~nm}$ [4], and penetration depth between 85 and $203 \mathrm{~nm}$ [4]. At a temperature much lower than its critical temperature, the surface resistance is considered to be dominated by the $\pi$-gap, and could potentially be much lower than that of $\mathrm{Nb}$ considering that $\mathrm{Nb}$ 's energy gap is $1.5 \mathrm{meV}$, and its critical temperature is $9.27 \mathrm{~K}$. This feature

\footnotetext{
*binping@bnl.gov

Published by the American Physical Society under the terms of the Creative Commons Attribution 4.0 International license. Further distribution of this work must maintain attribution to the author(s) and the published article's title, journal citation, and DOI.
}

also makes $\mathrm{MgB}_{2}$ attractive for use in high-performance multilayer film coatings proposed by Gurevich [9].

Various tests have been made to measure the surface resistance and reactance of $\mathrm{MgB}_{2}$ [10-13]. In reference [13], the authors used the surface impedance characterization (SIC) system [14] at Jefferson Lab (JLab) to test SRF properties of $\mathrm{MgB}_{2}$ at $7.4 \mathrm{GHz}$, with temperature ranges from $2.2 \mathrm{~K}$ to critical temperature. This work was distinguished from the previous works by higher resolution [13].

Mattis and Bardeen (MB) [15], and Abrikosov, Gor'kov, and Khalatnikov (AGK) [16], independently derived the theory to calculate the surface impedance of single band BCS superconductors. These two theories have the same expression in the low field limit. Computer codes developed by J. Halbritter based on AGK and the one developed by J.P. Turneaure based on $\mathrm{MB}$ are routinely used to calculate the $\mathrm{Nb}$ surface impedance for SRF applications [17]. However, there is yet a lack of a treatment of the surface impedance of two-band superconductors based on $\mathrm{MB}$ or AGK. In this paper, we further extend the MB theory to obtain the surface impedance of $\mathrm{MgB}_{2}$ and compare the simulated results with measured data [13].

\section{RF SURFACE IMPEDANCE OF A TWO-BAND SUPERCONDUCTOR}

Theoretical study of a two-band superconductor was initiated in 1959 [18]. The electrodynamic properties were extensively studied $[19,20]$. In this paper, we focus on the low field limit in which vortexes have not yet formed.

Following BCS theory [21], with temperature $T$ close to 0 , the probability of the state $(\boldsymbol{k} \uparrow,-\boldsymbol{k} \downarrow)$ being occupied by a pair of particles is $h_{k}$. With a finite $T$, single electrons start to appear. $f_{k}$ is defined as the probability that state $\boldsymbol{k} \uparrow$ or $\boldsymbol{- k} \downarrow$, with Bloch energy relative to the Fermi sea of $\varepsilon_{k}$, being occupied, which follows the Fermi-Dirac distribution. 
We use subscripts $d$ and $s$ to represent different bands in the superconductor, energy gap $A$ for band $s, B$ for band $d$, and $E_{k s}=\sqrt{\varepsilon_{k s}^{2}+A^{2}}, E_{k d}=\sqrt{\varepsilon_{k d}^{2}+B^{2}}$.

In order to calculate the SRF BCS surface impedance, one may start with the matrix elements of a single-particle scattering operator as in Refs. [15,21]. The scattering between $k s$ and $k^{\prime} s$, as well as the scattering between $k d$ and $k^{\prime} d$, should be the same as the case with single band gap. To calculate the effect brought by the scattering between $k d$ and $k^{\prime} s$, and also between $k s$ and $k^{\prime} d$, we list the matrix elements in Table I. In these columns, $h, h^{\prime}, f, f^{\prime}, E, E^{\prime}$ are used to simplify the expressions of $h_{k}, h_{k^{\prime}}, f_{k}, f_{k^{\prime}}, E_{k}$, and $E_{k^{\prime}}$, respectively. One may refer to Refs. [15,21] for more detail about this table.

While under rf field with angular frequency $\omega$, the photon energy $\hbar(\omega-i s)$ should be inserted into either the initial or the final state in Table I. Here a small positive parameter $s$, which will be set equal to zero in the final expression, has been introduced to obtain the real and imaginary part of surface impedance [15].

Based on the above analysis, the single-particle scattering operator, shown as Eq. (3.5) in Ref. [15] may be rewritten as,

$I(\omega, R, T)=V_{s s} N_{s} N_{s} I_{s s}(\omega, R, T)+V_{d d} N_{d} N_{d} I_{d d}(\omega, R, T)+\frac{V_{s d}+V_{d s}}{2} N_{s} N_{d} I_{s d}(\omega, R, T)+\frac{V_{s d}+V_{d s}}{2} N_{s} N_{d} I_{d s}(\omega, R, T)$

with

$$
\begin{aligned}
I_{s s}(\omega, R, T)= & -\pi i \int_{A-\hbar \omega}^{\infty}\left[1-2 f\left(E_{s 2}\right)\right]\left[g\left(E_{s}, E_{s 2}, A, A\right) \cos \left(\alpha \varepsilon_{s 2}\right)-i \sin \left(\alpha \varepsilon_{s 2}\right)\right] \mathrm{e}^{\mathrm{i} \alpha \varepsilon_{s}} d E_{s} \\
& +\pi i \int_{A}^{\infty}\left[1-2 f\left(E_{s}\right)\right]\left[g\left(E_{s}, E_{s 2}, A, A\right) \cos \left(\alpha \varepsilon_{s}\right)+i \sin \left(\alpha \varepsilon_{s}\right)\right] \mathrm{e}^{-\mathrm{i} \alpha \varepsilon_{s 2}} d E_{s} \\
I_{d d}(\omega, R, T)= & -\pi i \int_{B-\hbar \omega}^{\infty}\left[1-2 f\left(E_{d 2}\right)\right] \times\left[g\left(E_{d}, E_{d 2}, B, B\right) \cos \left(\alpha \varepsilon_{d 2}\right)-i \sin \left(\alpha \varepsilon_{d 2}\right)\right] \mathrm{e}^{\mathrm{i} \alpha \varepsilon_{d}} d E_{d} \\
& +\pi i \int_{B}^{\infty}\left[1-2 f\left(E_{d}\right)\right]\left[g\left(E_{d}, E_{d 2}, B, B\right) \cos \left(\alpha \varepsilon_{d}\right)+i \sin \left(\alpha \varepsilon_{d}\right)\right] \mathrm{e}^{-\mathrm{i} \alpha \varepsilon_{d 2}} d E_{d} \\
I_{s d}(\omega, R, T)= & -\pi i \int_{A-\hbar \omega}^{\infty}\left[1-2 f\left(E_{s 2}\right)\right]\left[g\left(E_{s}, E_{s 2}, A, B\right) \frac{\varepsilon_{s}}{\varepsilon_{s}^{*}} \cos \left(\alpha \varepsilon_{s 2}\right)-i \sin \left(\alpha \varepsilon_{s 2}\right)\right] \mathrm{e}^{\mathrm{i} \alpha \varepsilon_{s}^{*} d E_{s}} \\
& +\pi i \int_{A}^{\infty}\left[1-2 f\left(E_{s}\right)\right]\left[g\left(E_{s}, E_{s 2}, A, B\right) \frac{\varepsilon_{s 2}}{\varepsilon_{s 2}^{*}} \cos \left(\alpha \varepsilon_{s}\right)+i \sin \left(\alpha \varepsilon_{s}\right)\right] \mathrm{e}^{-\mathrm{i} \alpha \varepsilon_{s 2}^{*} d E_{s}} \\
I_{d s}(\omega, R, T)= & -\pi i \int_{B-\hbar \omega}^{\infty}\left[1-2 f\left(E_{d 2}\right)\right]\left[g\left(E_{d}, E_{d 2}, A, B\right) \frac{\varepsilon_{d}}{\varepsilon_{d}^{*}} \cos \left(\alpha \varepsilon_{d 2}\right)-i \sin \left(\alpha \varepsilon_{d 2}\right)\right] e^{i \alpha \varepsilon_{d}^{*} d E_{d}} \\
& +\pi i \int_{B}^{\infty}\left[1-2 f\left(E_{d}\right)\right]\left[g\left(E_{d}, E_{d 2}, A, B\right) \frac{\varepsilon_{d 2}}{\varepsilon_{d 2}^{*}} \cos \left(\alpha \varepsilon_{d}\right)+i \sin \left(\alpha \varepsilon_{d}\right)\right] e^{-i \alpha \varepsilon_{d 2}^{*} d E_{d}} \\
\text { and } E_{s 2}= & E_{s}+\hbar \omega, \quad E_{d 2}=E_{d}+\hbar \omega \quad \varepsilon_{s}^{*}=\sqrt{E_{s}^{2}-B^{2}}, \quad \varepsilon_{s 2}=\sqrt{E_{s 2}^{2}-A^{2},} \\
\varepsilon_{s 2}^{*}= & \sqrt{E_{s 2}^{2}-B^{2},} \quad \varepsilon_{d}^{*}=\sqrt{E_{d}^{2}-A^{2},} \quad \varepsilon_{d 2}=\sqrt{E_{d 2}^{2}-B^{2},} \quad \varepsilon_{d 2}^{*}=\sqrt{E_{d 2}^{2}-A^{2},} \\
g\left(E_{1}, E_{2}, \Delta_{1}, \Delta_{2}\right)= & \frac{E_{1} E_{2}+\Delta_{1} \Delta_{2}}{\varepsilon_{1} \varepsilon_{2}}, \quad \alpha=\frac{R}{\hbar p_{F}} . \quad
\end{aligned}
$$

$V_{s s}, V_{d d}, V_{s d}$, and $V_{d s}$ are the averaged interaction energies resulting from phonon emission and absorption by $s-s, d-d$, $s$ - $d$, and $d$-s processes, minus the corresponding shielded Coulomb interaction terms [18]. $N_{s}, N_{d}$ are the densities of states in the $s$ and $d$ bands near the Fermi level. The coefficient of $I_{s d}$ takes into account the single particle scattering from $s$ to $d^{\prime}$, as well as the scattering from $d^{\prime}$ to $s$. When $A=B$, (1) reduces to Eq. (3.5) in Ref. [15].
We use the $\mathrm{SRF}$ application of $\mathrm{MgB}_{2}$ at $7.5 \mathrm{GHz}$ range as an example. In this material the large energy gap is much bigger than the small energy gap. In this application $A-$ $\hbar \omega>B$ holds in the temperature range the SRF application is interested in, i.e., from $0 \mathrm{~K}$ to $0.1 \mathrm{~K}$ below the critical temperature.

To obtain the surface impedance, we use the expressions in Ref. [22], 
TABLE I. Matrix elements of single particle scattering operator (between $k s$ and $k^{\prime} d$ ).

\begin{tabular}{|c|c|c|c|c|c|c|c|}
\hline \multirow{2}{*}{\multicolumn{2}{|c|}{$\frac{\text { Wave functions }}{\text { Initial, } \psi_{i} \text { Final, } \psi_{f}}$}} & \multirow{2}{*}{\multicolumn{2}{|c|}{$\begin{array}{l}\text { Ground }(+) \text { or } \\
\text { excited }(-)\end{array}$}} & \multirow{3}{*}{$\begin{array}{c}\begin{array}{c}\text { Energy } \\
\text { difference }\end{array} \\
W_{i}-W_{f}\end{array}$} & \multirow{3}{*}{$\begin{array}{l}\text { Probability of } \\
\text { initial state }\end{array}$} & \multicolumn{2}{|c|}{ Matrix elements } \\
\hline & & & & & & \multirow{2}{*}{$\begin{array}{c}c_{k^{\prime} s \uparrow^{*}} c_{k d \uparrow} \text { or } \\
c_{-k^{\prime} s \downarrow}{ }^{*} c_{k d \uparrow}\end{array}$} & \multirow{2}{*}{$\begin{array}{c}c_{-k d \downarrow}{ }^{*} c_{-k^{\prime} s \downarrow} \text { or } \\
-c_{-k d \downarrow}{ }^{*} c_{k^{\prime} s \uparrow}\end{array}$} \\
\hline$k s k^{\prime} d$ & $k s k^{\prime} d$ & $k s$ & $k^{\prime} d$ & & & & \\
\hline \multicolumn{2}{|c|}{ (a) } & + & + & $E_{s}-E_{d^{\prime}}$ & $1 / 2 s_{s}\left(1-s_{d^{\prime}}-p_{d^{\prime}}\right)$ & {$\left[\left(1-h_{s}\right)\left(1-h_{d^{\prime}}\right)\right]^{1 / 2}$} & $-\left(h_{s} h_{d^{\prime}}\right)^{1 / 2}$ \\
\hline X0 00 & $00 \mathrm{X} 0$ & - & - & $-E_{s}+E_{d^{\prime}}$ & $1 / 2 s_{s} p_{d^{\prime}}$ & $\left(h_{s} h_{d^{\prime}}\right)^{1 / 2}$ & $-\left[\left(1-h_{s}\right)\left(1-h_{d^{\prime}}\right)\right]^{1 / 2}$ \\
\hline \multirow[t]{2}{*}{$\mathrm{X} 0 \mathrm{XX}$} & XX X0 & + & - & $E_{s}+E_{d^{\prime}}$ & $1 / 2 s_{s} p_{d^{\prime}}$ & $-\left[\left(1-h_{s}\right) h_{d^{\prime}}\right]^{1 / 2}$ & $-\left[h_{s}\left(1-h_{d^{\prime}}\right)\right]^{1 / 2}$ \\
\hline & & - & + & $-E_{s}-E_{d^{\prime}}$ & $1 / 2 s_{s}\left(1-s_{d^{\prime}}-p_{d^{\prime}}\right)$ & $-\left[h_{s}\left(1-h_{d^{\prime}}\right)\right]^{1 / 2}$ & $-\left[\left(1-h_{s}\right) h_{d^{\prime}}\right]^{1 / 2}$ \\
\hline \multicolumn{2}{|c|}{ (b) } & + & + & $-E_{s}+E_{d^{\prime}}$ & $1 / 2 s_{d^{\prime}}\left(1-s_{s}-p_{s}\right)$ & $\left(h_{s} h_{d^{\prime}}\right)^{1 / 2}$ & $-\left[\left(1-h_{s}\right)\left(1-h_{d^{\prime}}\right)\right]^{1 / 2}$ \\
\hline XX 0X & $0 \mathrm{XXX}$ & - & - & $E_{s}-E_{d^{\prime}}$ & $1 / 2 s_{d^{\prime}} p_{s}$ & {$\left[\left(1-h_{s}\right)\left(1-h_{d^{\prime}}\right)\right]^{1 / 2}$} & $-\left(h_{s} h_{d^{\prime}}\right)^{1 / 2}$ \\
\hline \multirow[t]{2}{*}{$000 \mathrm{X}$} & $0 \times 00$ & + & - & $-E_{s}-E_{d^{\prime}}$ & $1 / 2 s_{d^{\prime}}\left(1-s_{s}-p_{s}\right)$ & {$\left[h_{s}\left(1-h_{d^{\prime}}\right)\right]^{1 / 2}$} & {$\left[\left(1-h_{s}\right) h_{d^{\prime}}\right]^{1 / 2}$} \\
\hline & & - & + & $E_{s}+E_{d^{\prime}}$ & $1 / 2 s_{d^{\prime}} p_{s}$ & {$\left[\left(1-h_{s}\right) h_{d^{\prime}}\right]^{1 / 2}$} & {$\left[h_{s}\left(1-h_{d^{\prime}}\right)\right]^{1 / 2}$} \\
\hline & c) & + & + & $E_{s}+E_{d^{\prime}}$ & $1 / 4 s_{s} s_{d^{\prime}}$ & {$\left[\left(1-h_{s}\right) h_{d^{\prime}}\right]^{1 / 2}$} & {$\left[h_{s}\left(1-h_{d^{\prime}}\right)\right]^{1 / 2}$} \\
\hline \multirow[t]{3}{*}{$\mathrm{X} 00 \mathrm{X}$} & $00 \mathrm{XX}$ & - & - & $-E_{s}-E_{d^{\prime}}$ & $1 / 4 s_{s} s_{d^{\prime}}$ & $-\left[h_{s}\left(1-h_{d^{\prime}}\right)\right]^{1 / 2}$ & $-\left[\left(1-h_{s}\right) h_{d^{\prime}}\right]^{1 / 2}$ \\
\hline & XX 00 & + & - & $E_{s}-E_{d^{\prime}}$ & $1 / 4 s_{s} s_{d^{\prime}}$ & {$\left[\left(1-h_{s}\right)\left(1-h_{d^{\prime}}\right)\right]^{1 / 2}$} & $-\left(h_{s} h_{d^{\prime}}\right)^{1 / 2}$ \\
\hline & & - & + & $-E_{s}-E_{d^{\prime}}$ & $1 / 4 s_{s} s_{d^{\prime}}$ & $-\left(h_{s} h_{d^{\prime}}\right)^{1 / 2}$ & {$\left[\left(1-h_{s}\right)\left(1-h_{d^{\prime}}\right)\right]^{1 / 2}$} \\
\hline \multicolumn{2}{|c|}{ (d) } & + & + & $-E_{s}-E_{d^{\prime}}$ & $\left(1-s_{s}-p_{s}\right)\left(1-s_{d^{\prime}}-p_{d^{\prime}}\right)$ & {$\left[h_{s}\left(1-h_{d^{\prime}}\right)\right]^{1 / 2}$} & {$\left[\left(1-h_{s}\right) h_{d^{\prime}}\right]^{1 / 2}$} \\
\hline XX 00 & $0 \times \times 0$ & - & - & $E_{s}+E_{d^{\prime}}$ & $p_{s} p_{d^{\prime}}$ & $-\left[\left(1-h_{s}\right) h_{d^{\prime}}\right]^{1 / 2}$ & $-\left[h_{s}\left(1-h_{d^{\prime}}\right)\right]^{1 / 2}$ \\
\hline \multirow[t]{2}{*}{$00 \mathrm{XX}$} & & + & - & $-E_{s}+E_{d^{\prime}}$ & $\left(1-s_{s}-p_{s}\right) p_{d^{\prime}}$ & $-\left(h_{s} h_{d^{\prime}}\right)^{1 / 2}$ & {$\left[\left(1-h_{s}\right)\left(1-h_{d^{\prime}}\right)\right]^{1 / 2}$} \\
\hline & & - & + & $E_{s}-E_{d^{\prime}}$ & $p_{s}\left(1-s_{d^{\prime}}-p_{d^{\prime}}\right)$ & {$\left[\left(1-h_{s}\right)\left(1-h_{d^{\prime}}\right)\right]^{1 / 2}$} & $-\left(h_{s} h_{d^{\prime}}\right)^{1 / 2}$ \\
\hline
\end{tabular}

$$
Z=i \pi \omega \mu_{0}\left\{\int_{0}^{\infty} \ln \left[1+K(p) / p^{2}\right] d p\right\}^{-1}
$$

where

$$
\begin{aligned}
K(p)= & \frac{-3}{4 \pi \hbar v_{F} \lambda_{L}^{2}(0)} \int_{0}^{\infty} \int_{-1}^{1} e^{i p R u} e^{-\frac{R}{T}} \\
& \times\left(1-u^{2}\right) I(\omega, R, T) d u d R
\end{aligned}
$$

with $\lambda_{L}$ London penetration depth, and $K(p)$ the component that connects the Fourier components of current and vector potential [22].

One now obtains an analytical expression for the surface impedance by incorporating (1) into (3), then into (2).

A Mathematica ${ }^{\mathrm{TM}}$ program has been developed to calculate this integral. In calculations below we use the following parameters as a reference: $A_{0}=7.1 \mathrm{meV}$, $B_{0}=2.3 \mathrm{meV} \quad[8], \quad T_{c}=39.5 \mathrm{~K} \quad[4], \quad l=40 \mathrm{~nm}$, $\lambda_{L}=100 \mathrm{~nm}$ [4], Debye temperature $=884 \mathrm{~K}$ [23] and Fermi velocity $=4.7 \times 10^{5} \mathrm{~m} / \mathrm{s}$ [24], with outside conditions $T=2 \mathrm{~K}$ and frequency at $7.5 \mathrm{GHz}$. The interaction factors are $V_{s d}=0.119, \quad V_{d s}=0.09, \quad V_{s s}=0.81$, $V_{d d}=0.285$, [25] and $N_{s}=1, N_{d}=1.3$ [26,27].

Using the parameters above, the surface impedance of $\mathrm{MgB}_{2}$ is calculated and compared with the experimental results in [13], with two samples having a $200 \mathrm{~nm}$ thick
$\mathrm{MgB}_{2}$ layer on sapphire $\left(\mathrm{MgB}_{2}-200-\mathrm{I}\right.$ and $\left.\mathrm{MgB}_{2}-200-\mathrm{II}\right)$, and one sample with $350 \mathrm{~nm}$ thick $\mathrm{MgB}_{2}$ on sapphire $\left(\mathrm{MgB}_{2}-350\right)$. The surface resistance data and theory are shown in Fig. 1(a). Since the SIC system measures the changes of penetration depth (relative penetration depth), the measured and simulated results of relative penetration depth are shown in Fig. 1(b). The surface resistance data and theory agree above $\sim 7 \mathrm{~K}$. Below that temperature, the experimental surface resistance levels out and appears dominated by some residual resistance mechanism not included in the theory.

For comparison, the calculation results of the two-band analysis are compared with the results for single band superconductors. With all other parameters the same as the above, surface impedance at different energy gaps, at $7.1 \mathrm{meV}$ ( $\sigma$-gap), and $2.3 \mathrm{meV}$ ( $\pi$-gap) were calculated; results are shown in Fig. 1. As expected, the surface impedance of a two-band superconductor is greater than a single band superconductor with a $\sigma$-gap, and is smaller than that with a $\pi$-gap. The surface resistance is clearly dominated by the smaller $\pi$-gap [27,28]. The penetration depth can also be fitted by an effective gap [28], shown in Fig. 2 in Ref. [13] at $3.7 \mathrm{meV}$. In the dirty limit with $l$ close to 0 , calculation shows the contribution from interband scattering [ $s d$ and $d s$ terms in Eq. (1)] is orders of magnitude lower than those from intra-band scattering [ss and $d d$ terms in Eq. (1)]. This agrees with Ref. [27] and references therein. 

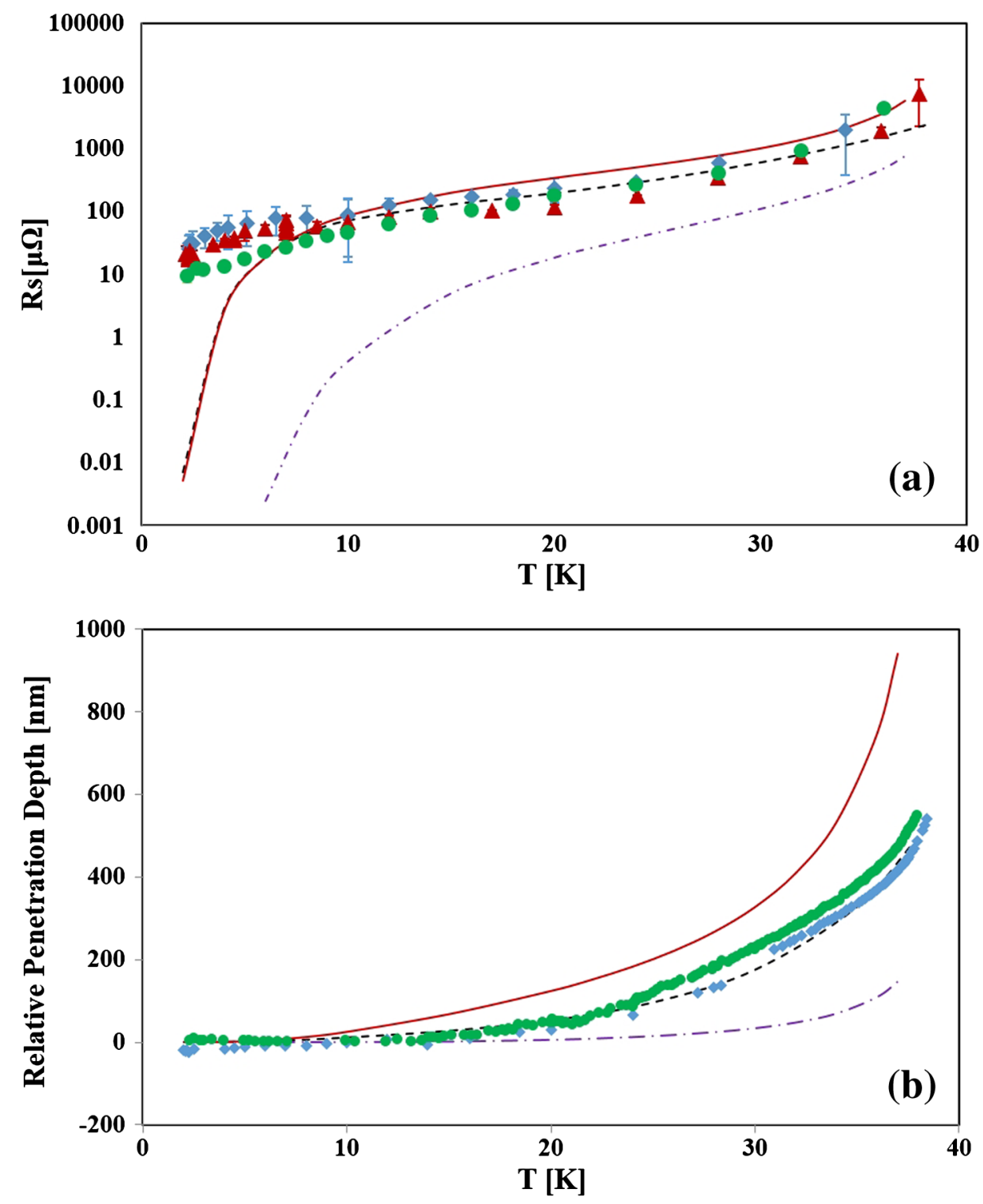

FIG. 1. (a) Surface resistance, and (b) relative penetration depth of $\mathrm{MgB}_{2}$. Blue diamond $\mathrm{MgB}_{2}-200-\mathrm{I}$, red triangle $\mathrm{MgB} 2-200-\mathrm{II}$, green circle $\mathrm{MgB}_{2}-350$, black dotted line is the two-gap analysis, red solid line is single $\pi$-gap calculation, and purple dash-dot line is single $\sigma$-gap calculation.

\section{CONCLUSION AND DISCUSSION}

With recent activities that are pushing the performance of $\mathrm{Nb}$ SRF cavities to their theoretical limits, the necessity of finding alternative materials for SRF application becomes more important. $\mathrm{MgB}_{2}$, as the conventional superconductor with highest critical temperature, is a favorite candidate. By applying the theory of a two-band superconductor to the MB theory, we calculated the surface impedance of $\mathrm{MgB}_{2}$. The results agree well with previously published experimental results.

Among all type I superconductors, $\mathrm{MgB}_{2}$ has the highest critical temperature and thus is attractive for higher temperature SRF applications with corresponding lower cryogenic power demands, perhaps within range of closed-cycle cryocooling systems. In the sample measurement reported in Fig. 1, the residual resistance dominates at temperatures below $7 \mathrm{~K}$. The mechanism(s) for these losses are yet unknown. Perhaps recent activities on $\mathrm{Nb}$ cavities $[29,30]$ could be studied with $\mathrm{MgB}_{2}$ thin films to further elucidate and suppress the residual resistance. Significant $R \& D$ is needed to make $\mathrm{MgB}_{2}$ suitable for SRF applications at viable accelerating field gradients. First beneficial applications in accelerator systems could perhaps be in low-field regions that are at intermediate temperature, e.g., rf couplers, beamlines, or beamline bellows, which rely on bulk conduction cooling or use return liquid or gas helium from other SRF or superconducting magnet components. 


\section{ACKNOWLEDGMENTS}

This work is supported by Brookhaven Science Associates, LLC under U.S. DOE Contract No. DEAC02-98CH10886, and by Jefferson Science Associates, LLC under U.S. DOE Contract No. DE-AC05-06OR23177. The U.S. Government retains a nonexclusive, paid-up, irrevocable, world-wide license to publish or reproduce this manuscript for U.S. Government purposes.

[1] A. Grassellino, A. Romanenko, D. Sergatskov, O. Melnychuk, Y. Trenikhina, A. Crawford, A. Rowe, M. Wong, T. Khabiboulline, and F. Barkov, Nitrogen and argon doping of niobium for superconducting radio frequency cavities: A pathway to highly efficient accelerating structures, Supercond. Sci. Technol. 26, 102001 (2013).

[2] B. P. Xiao, C. E. Reece, and M. J. Kelley, Superconducting surface impedance under radiofrequency field, Phys. C 490, 26 (2013).

[3] J. Nagamatsu, N. Nakagawa, T. Muranaka, Y. Zenitani, and J. Akimitsu, Superconductivity at $39 \mathrm{~K}$ in magnesium diboride, Nature (London) 410, 63 (2001).

[4] C. Buzea and T. Yamashita, Review of the superconducting properties of $\mathrm{MgB}_{2}$, Supercond. Sci. Technol. 14, R115 (2001).

[5] R. S. Gonnelli, D. Daghero, G. A. Ummarino, V. A. Stepanov, J. Jun, S. M. Kazakov, and J. Karpinski, Direct Evidence for Two-Band Superconductivity in $\mathrm{MgB}_{2}$ Single Crystals from Directional Point-Contact Spectroscopy in Magnetic Fields, Phys. Rev. Lett. 89, 247004 (2002).

[6] H. J. Choi, D. Roundy, H. Sun, M. L. Cohen, and S. G. Louie, The origin of the anomalous superconducting properties of $\mathrm{MgB}_{2}$, Nature (London) 418, 758 (2002).

[7] I. I. Mazin and V.P. Antropov, Electronic structure, electron-phonon coupling, and multiband effects in $\mathrm{MgB}_{2}$, Phys. C 385, 49 (2003).

[8] M. Iavarone, G. Karapetrov, A. E. Koshelev et al., Two-Band Superconductivity in $\mathrm{MgB}_{2}$, Phys. Rev. Lett. 89, 187002 (2002).

[9] A. Gurevich, Enhancement of rf breakdown field of superconductors by multilayer coating, Appl. Phys. Lett. 88, 012511 (2006).

[10] A. G. Zaitsev, R. Schneider, R. Hott et al., Microwave properties of $\mathrm{MgB}_{2}$ thin films prepared in situ by thermal evaporation combined with sputtering, J. Phys. Conf. Ser. 43, 309 (2006).

[11] B. B. Jin, N. Klein, W. N. Kang, H.-J. Kim, E.-M. Choi, S.-I. Lee, T. Dahm, and K. Maki, Energy gap, penetration depth and surface resistance of $\mathrm{MgB}_{2}$ thin films determined by microwave resonator measurements, Phys. Rev. B 66, 104521 (2002).

[12] N. Klein, B. B. Jin, R. Wordenweber, P. Lahl, W. N. Kang, H.-J. Kim, E.-M. Choi, S.-I. Lee, T. Dahm, and K. Maki, Microwave properties of $\mathrm{MgB}_{2}$ thin films, IEEE Trans. Appl. Supercond. 13, 3253 (2003).

[13] B. P. Xiao, X. Zhao, J. Spradlin, C. E. Reece, M. J. Kelley, T. Tan, and X. X. Xi, Surface impedance measurements of single crystal $\mathrm{MgB}_{2}$ films for radiofrequency superconductivity applications, Supercond. Sci. Technol. 25, 095006 (2012).

[14] B. P. Xiao, C. E. Reece, H. L. Phillips, R. L. Geng, H. Wang, F. Marhauser, and M. J. Kelley, Radio frequency surface impedance characterization system for superconducting samples at 7.5 GHz, Rev. Sci. Instrum. 82, 056104 (2011).

[15] D. C. Mattis and J. Bardeen, Theory of the anomalous skin effect in normal and superconducting metals, Phys. Rev. 111, 412 (1958).

[16] A. Abrikosov, L. Gor'kov, and I. Khalatnikov, A superconductor in a high frequency field, Sov. Phys. JETP 35, 182 (1959).

[17] J. P. Turneaure, J. Halbritter, and H. A. Schwettman, The surface impedance of superconductors and normal conductors: The Mattis-Bardeen theory, J. Supercond. 4, 341 (1991).

[18] H. Suhl, B. T. Matthias, and L. R. Walker, BardeenCooper-Schrieffer Theory of Superconductivity in the Case of Overlapping Bands, Phys. Rev. Lett. 3, 552 (1959).

[19] E. Babaev, J. Carlström, M. Silaev et al., Type-1.5 superconductivity in multicomponent systems, Phys. C 533, 20 (2017).

[20] M. Silaev, T. Winyard, and E. Babaev, Non-London electrodynamics in a multiband London model: Anisotropy-induced nonlocalities and multiple magnetic field penetration lengths, Phys. Rev. B 97, 174504 (2018).

[21] J. Bardeen, L. N. Cooper, and J. R. Schrieffer, Theory of superconductivity, Phys. Rev. 108, 1175 (1957).

[22] P. B. Miller, Surface impedance of superconductors, Phys. Rev. 118, 928 (1960).

[23] Z. F. Wei, G. C. Che, F. M. Wang, W. C. Wang, M. He, and X. L. Chen, Debye temperature of $\mathrm{MgB}_{2}$, Mod. Phys. Lett. B 15, 1109 (2001).

[24] A. Kohen and G. Deutscher, Symmetry and temperature dependence of the order parameter in $\mathrm{MgB}_{2}$ from point contact measurements, Phys. Rev. B 64, 060506 (2001).

[25] A. Gurevich, Enhancement of the upper critical field by nonmagnetic impurities in dirty two-gap superconductors, Phys. Rev. B 67, 184515 (2003).

[26] K. D. Belashchenko, M. van Schilfgaarde, and V.P. Antropov, Coexistence of covalent and metallic bonding in the boron intercalation superconductor $\mathrm{MgB}_{2}$, Phys. Rev. B 64, 092503 (2001).

[27] B. B. Jin, T. Dahm, A. I. Gubin, E.-M. Choi, H. J. Kim, S.-I. Lee, W. N. Kang, and N. Klein, Anomalous Coherence Peak in the Microwave Conductivity of c-Axis Oriented $\mathrm{MgB}_{2}$ Thin Films, Phys. Rev. Lett. 91, 127006 (2003).

[28] G. Ghigo, D. Botta, A. Chiodoni, L. Gozzelino, R. Gerbaldo, F. Laviano, E. Mezzetti, E. Monticone, and C. Portesi, Effective gap at microwave frequencies in $\mathrm{MgB}_{2}$ thin films with strong interband scattering, Phys. Rev. B 71, 214522 (2005).

[29] A. Romanenko, A. Grassellino, A. C. Crawford, D. A. Sergatskov, and O. Melnychuk, Ultra-high quality factors in superconducting niobium cavities in ambient magnetic fields up to $190 \mathrm{mG}$, Appl. Phys. Lett. 105, 234103 (2014).

[30] A. Romanenko, A. Grassellino, O. Melnychuk, and D. A. Sergatskov, Dependence of the residual surface resistance of superconducting radio frequency cavities on the cooling dynamics around Tc, J. Appl. Phys. 115, 184903 (2014). 